\title{
CRISPR: A Technical Breakthrough for Tomato Research $^{1}$
}

\author{
Tong Geon Lee ${ }^{2}$
}

\section{Introduction}

You probably have heard of CRISPR (pronounced "crisper"). This contemporary biological technology is one of the latest and most controversial issues today in terms of ethical, socioeconomic, and regulatory perspectives. Unsurprisingly, this technology has not only attracted the interests of scientists in public and private sectors, but also those of the general public as well. Many crops, including tomato, stand to benefit from applications of CRISPR technology, and growers/producers and consumers wait with anticipation to see what improvements will be made. In this article, three topics are discussed: 1 ) why tomato researchers are interested in CRISPR, 2) what tomato traits have been targeted by CRISPR, and 3) potential applications of CRISPR for tomato genetics and breeding. This work is intended for both non-scientific and scientific audiences.

\section{Why Tomato Researchers are Interested in CRISPR}

In the 1980s, biologists discovered that some bacteria use a unique system for protection against viral attacks. This system actually targeted the viruses' DNA sequence, making changes to the sequence in order to defeat viruses. Scientists named the system CRISPR (l)

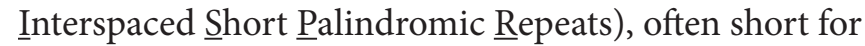
CRISPR-Cas (ㄷISPR-associated). Researchers around the world quickly adopted this system to edit the DNA sequences of interest in viruses, bacteria, animals, and plants. Tomato geneticists and breeders have likewise become interested in this technology, because changing a gene's DNA sequence could be a valuable tool for geneticists to study traits and for breeders to make crop improvement.

The conventional breeding paradigm has been enormously successful in creating cultivars with improved quality and productivity. Nonetheless, difficulties have often been encountered as breeders have sought to identify and introduce new traits. One example, from many such cases, is the development of nematode-resistant tomatoes. During the past 70 years, there was only one nematode resistance gene, $M i$, which has been successfully introgressed (meaning the gene was permanently incorporated from one line to another by breeding) into commercially-acceptable varieties. Yet, US tomato producers are further challenged because the Mi gene is continuing to lose efficacy. What is the solution? Conventional breeding is obviously a longterm investment, especially when a novel resistance gene, such as $M i$, is introduced from a wild tomato.

Basically, conventional breeding is not considered a tool of precision. It essentially involves a "reshuffling of the deck" of genes that exist between the parents, followed by multiple cycles of selection for one or a few genes of interest or new favorable gene combinations. In contrast, CRISPR has the proven ability to 1) precisely change DNA sequence of a gene and 2) minimize unwanted changes to other DNA sequences besides the one of interest. These characteristics

1. This document is HS1314, one of a series of the Horticultural Sciences Department, UF/IFAS Extension. Original publication date February 2018. Visit the EDIS website at http://edis.ifas.ufl.edu.

2. Tong Geon Lee, assistant professor, Department of Horticultural Sciences; UF/IFAS Gulf Coast Research and Education Center, Wimauma, FL 33598.

The Institute of Food and Agricultural Sciences (IFAS) is an Equal Opportunity Institution authorized to provide research, educational information and other services only to individuals and institutions that function with non-discrimination with respect to race, creed, color, religion, age, disability, sex, sexual orientation, marital status, national origin, political opinions or affiliations. For more information on obtaining other UF/IFAS Extension publications, contact your county's UF/IFAS Extension office. 
have the potential to significantly speed up the breeding cycle (Figure 1).

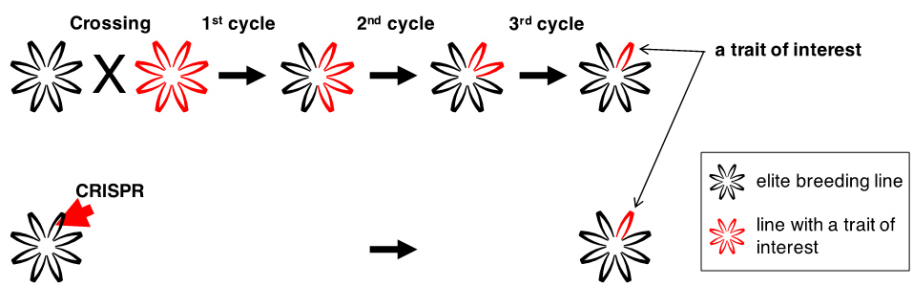

Figure 1. A conceptual scheme for CRISPR when compared to conventional crossing method to introgress a trait of interest to an elite breeding line. Upper diagram: an introgression of a trait of interest in the conventional breeding paradigm through crossing, which requires years of breeding efforts to eliminate negative horticultural traits. Lower diagram: CRISPR-mediated introgression of a trait, which delivers a trait of interest to an elite breeding line.

CRISPR can also provide advantages over traditional approaches to genetics. For example, geneticists have studied the function of genes by using mutations (changes to the DNA sequence). However, such mutations have historically required much time and labor to develop. In contrast, CRISPR generally has a short development time and low equipment and labor costs.

\section{What tomato traits have been targeted by CRISPR?}

Recently, CRISPR has been integrated into a number of tomato genetics research projects to create various gene-edited tomatoes. Diverse traits have been targeted by this system, and research results have been published in peer-reviewed scientific journals (Table 1). Recent progress in understanding the mechanism of flowering-related traits, notably of the jointless pedicel trait (Soyk et al. 2017a), was aided by CRISPR. Specifically, CRISPR was used to inactivate a gene controlling the jointed pedicel trait, which resulted in the conversion to jointless pedicels. Because the jointless pedicel trait is important for stem-free harvesting of fruit, this example of gene-editing may have direct application toward the UF/IFAS tomato breeding program's goal to develop mechanically harvestable tomatoes.

\section{The Future of Gene-Edited}

\section{Tomatoes}

Nonetheless, despite the potential of CRISPR for tomato improvement, considerations are required for this technology to be fully applied to future tomato research. The ability to knock out genes in a cost- or time-effective way is the main continuing advantage of CRISPR over existing methods for the discovery of gene function. While, in principle, it is possible that this technology can be used to introduce novel gene(s) (e.g., allele swapping), CRISPR applications are still generally limited to cutting DNA sequences and inactivating genes. Advancements in this technology could lead to the ability to swap alleles or introduce novel genes that would broaden germplasm resources, but also limit the introduction of unwanted genes (linkage drag).

CRISPR-driven traits are eventually likely to have a large impact on commercially-acceptable backgrounds, but have primarily been developed for the purpose of general genetic studies. This is presently evidenced by the fact that most of the work done to date has been in small numbers of model tomatoes such as Micro-Tom and M82 (Table 1). Therefore, to apply the full power of CRISPR to tomato improvement, it is first necessary either to advance such traits into the genetic background of commercially-acceptable tomatoes or to repeat the gene-editing process using commerciallyacceptable tomatoes instead of model ones.

A final issue that must be considered is how this technology may be regulated. There will likely be no restrictions on the use of CRISPR for plant genetics or other research projects for which the gene-edited plants are not destined for commercialization. But there is considerable uncertainty whether CRISPR-driven gene-edited plants will be legally classified as genetically modified organisms (GMOs), which are subject to the USDA regulatory system. In the US, a GMO is defined as an organism that has a new combination of genetic material (e.g., DNA) that could not have occurred naturally. As currently used, CRISPR produces changes in plant's genetic material, but it conceptually does not introduce or create new combinations of genetic material (e.g., a mix of host and non-host DNAs); it is important to distinguish between the use of traditional genetic engineering methods (excluding gene-editing technologies) for modifying genetic material (GMOs via inserting foreign gene sequences) and the use of current CRISPR (where this system edits sequences of the host). In addition, conventional plant crossing methods can be used to remove any unwanted remains of non-host DNA. Therefore, CRISPR-driven tomato improvements may be fundamentally different in concept to GMOs. Two examples are CRISPR-driven, gene-edited waxy corn (DuPont Pioneer) and white mushroom (Pennsylvania State University), which the USDA will not subject to the same rules as traditional GMOs.

\section{Conclusion}

Scientists are enjoying a new era in research, using CRISPR technology to perform genetic studies in tomato with the utmost specificity and precision. Yet, this contemporary 
tool has limitations that may limit this system's applicability for tomato improvement. Nevertheless, CRISPR technology continues to evolve, and further advances to the system will create new opportunities for improvements that both tomato researchers and the public will share. But how extensively CRISPR is used for developing new tomato varieties remains a source of debate among participants. The next few years will be an exciting time, as the utilization of CRISPR in plant genetics and breeding expands further.

\section{References}

Brooks, C., V. Nekrasov, Z. B. Lippman, and J. Van Eck. 2014. "Efficient gene editing in tomato in the first generation using the clustered regularly interspaced short palindromic repeats/CRISPR-associated 9 system." Plant Physiol. 166(3):1292-1297.

Čermák, T., N. J. Baltes, R. Čegan, Y. Zhang, and D. F. Voytas. 2015. "High-frequency, precise modification of the tomato genome." Genome Biol. 16:232.

Jacobs. T. B., N. Zhang, D. Patel, and G. B. Martin. 2017. "Generation of a collection of mutant tomato lines using pooled CRISPR libraries.” Plant Physiol. 174(4):2023-2037.

Nekrasov, V., C. Wang, J. Win, C. Lanz, D. Weigel, and S. Kamoun. 2017. "Rapid generation of a transgene-free powdery mildew resistant tomato by genome deletion." Sci Rep. 7(1):482.

Pan, C., L. Ye, L. Qin, X. Liu, Y. He, J. Wang, L. Chen, and G. Lu. 2016. "CRISPR/Cas9-mediated efficient and heritable targeted mutagenesis in tomato plants in the first and later generations." Sci Rep. 6:24765.

Ron, M., K. Kajala, G. Pauluzzi, D. Wang, M. A. Reynoso, K. Zumstein, J. Garcha, S. Winte, H. Masson, S. Inagaki, F. Federici, N. Sinha, R. B. Deal, J. Bailey-Serres, and S. M. Brady. 2014. "Hairy root transformation using Agrobacterium rhizogenes as a tool for exploring cell type-specific gene expression and function using tomato as a model." Plant Physiol. 166(2):455-469.

Soyk, S., Z. H. Lemmon, M. Oved, J. Fisher, K. L. Liberatore, S. J. Park, A. Goren, K. Jiang, A. Ramos, E. van der Knaap, J. Van Eck, D. Zamir, Y. Eshed, and Z. B. Lippman. 2017a. "Bypassing negative epistasis on yield in tomato imposed by a domestication gene." Cell. 169(6):1142-1155. e12.
Soyk, S., N. A. Müller, S. J. Park, I. Schmalenbach, K. Jiang, R. Hayama, L. Zhang, J. Van Eck, J. M. Jiménez-Gómez, and Z. B. Lippman. 2017b. "Variation in the flowering gene SELF PRUNING $5 G$ promotes day-neutrality and early yield in tomato." Nat Genet. 49(1):162-168.

Ueta, R., C. Abe, T. Watanabe, S. S. Sugano, R. Ishihara, H. Ezura, Y. Osakabe, and K. Osakabe. 2017. "Rapid breeding of parthenocarpic tomato plants using CRISPR/Cas9.” Sci Rep. 7(1):507.

Xu, C., K.L. Liberatore, C. A. MacAlister, Z. Huang, Y. H. Chu, K. Jiang, C. Brooks, M. Ogawa-Ohnishi, G. Xiong, M. Pauly, J. Van Eck, Y. Matsubayashi, E. van der Knaap, and Z. B. Lippman. 2015. "A cascade of arabinosyltransferases controls shoot meristem size in tomato." Nat Genet. 47(7):784-792.

Xu, C., S. J. Park, J. Van Eck, and Z. B. Lippman. 2016. "Control of inflorescence architecture in tomato by BTB/POZ transcriptional regulators." Genes Dev. 30(18):2048-2061. 
Table 1. Current applications of CRISPR to tomato.

\begin{tabular}{|l|l|l|}
\hline \multicolumn{1}{|c|}{ Target traits } & \multicolumn{1}{|c|}{ Tomato lines used for experiment } & \multicolumn{1}{c|}{ Scientific publications } \\
\hline leaf morphology & M82 & Brooks et al. 2014 \\
\hline root morphology & M82 & Ron et al. 2014 \\
\hline leaf/stem color & Micro-Tom & Čermák et al. 2015 \\
\hline shoot meristem size & M82 & Xu et al. 2015 \\
\hline albino phenotype & Micro-Tom & Pan et al. 2016 \\
\hline inflorescence architecture & M82 & Xu et al. 2016 \\
\hline canonical disease resistance-related & M82 & Jacobs et al. 2017 \\
\hline powdery mildew resistance & Moneymaker & Nekrasov et al. 2017 \\
\hline jointless pedicels & M82 & Soyk et al. 2017a \\
\hline flowering habit & M82, Sweet 100 & Soyk et al. 2017b \\
\hline parthenocarpy (fruit production without fertilization) & Ailsa Craig, Micro-Tom & Ueta et al. 2017 \\
\hline
\end{tabular}

